\title{
Hip Joint Osteoradionecrosis following Pelvic Radiotherapy: A Report of Two Cases
}

\author{
Rajaallah Abdessamad*, Sidi Elhacen, Messoudi Abdeljabbar, Garnaoui Hatim and Garch Abdelhak* \\ Department of Orthopedics, HASSAN II Casablanca University, Moroccco
}

Submission: January 05, 2021; Published: January 22, 2021

*Corresponding author: Rajaallah Abdessamad, Department of Orthopedics, HASSAN II Casablanca University, Res Les Collines Tr 3 Immeuble D Appart 15 Sidi Maarouf Casablanca, Moroccco

\begin{abstract}
Radiotherapy represents a highly effective and curative treatment modality for the treatment of pelvic malignancies and for metastatic bone disease. One of the most severe and challenging long-term complication in radiotherapy is osteonecrosis. These changes may be deleterious effects on osseointegration and provide a challenge for long-term implant stability in Total Hip Arthroplasty patients that remains a challenge because of high rates of early failure of traditional implants. The addition of a Kerboull reinforcement cross to the reconstruction reduce the risk of loosening of the acetabular component by giving elasticity to the implant, decreasing the stress applied to the peri-acetabular bone, and allows fixation over a large surface area. There are very few cases of radiation-induced hip necrosis described in the literature. We report two cases of hip joint necrosis with an acetabular protrusion and femoral head deformities, after a therapeutic pelvic radiation for cervical cancer managed by a total hip arthroplasty, by a cemented femoral and acetabular component with the addition of a Kerboull reinforcement cross.
\end{abstract}

Keywords: Hip joint; Radiotherapy; Osteoradionecrosis ; Total hip arthroplasty

\section{Introduction}

Pelvic irradiation is commonly used in the treatment of pelvic malignancies and for metastatic bone disease [1]. Modern irradiation planning techniques enable very accurate dose distribution and precise beam delivery. However, Frequent osseous complications are summarized as radiation-induced reactions. One of the most severe and challenging long-term complication in radiotherapy is osteonecrosis [2,3]. There are very few documented cases of radiation-induced hip joint necrosis in the literature [4]. We report two cases of hip joint necrosis with an acetabular protrusion and femoral head deformities, after a therapeutic pelvic radiation.

\section{Case Report}

\section{Case 1}

A 53-year-old female, suffered from cervical cancer, the patient was treated with radical hysterectomy and postoperative chemotherapy and radiotherapy two years before presentation. The exact dose distribution within the pelvis is not available. One years after therapy, the patient started to feel pain in her right hip and the inguinal region increasing with movement, which had been progressive and became worse recently. and had no history of fall, trauma or osteoarthritis in the left hip. She had no fever, night sweats, or weight loss. Examination of the left hip revealed painful movements with restriction in the range of motion, and a shortening of the lower left limb of $1 \mathrm{~cm}$. There were no inflammatory signs, and the rest of the general examination was normal.

A radiograph of the pelvis showed an acetabular osteolysis with protrusion, destruction of the left femoral head and pathological central dislocation of the hip (Figure 1a). Computed Tomography (CT) revealed irregular Osteolytic process of the acetabulum with an ill-defined border and femoral head destruction, with intra-articular bone fragments (Figure 1b). Magnetic Resonance Imaging (MRI) of the pelvis revealed a remodeling of the left hip in T1 hyposignal and hypersignal in T2 and STIR, moderately enhanced by the contrast product and severe destruction of the femoral head, suggestive of osteonecrosis of the hip (Figure 2). The bone scan revealed increased uptake in the left acetabulum and femoral head, but there were no other suspicious areas of increased uptake to suggest metastases (Figure 3). To eliminate the diagnosis of bone metastasis or Septic arthritis of the hip joint, a CT-guided biopsy was arranged showing Chronic inflammatory remodeling without evidence of malignancy, with signs related to bone avascular necrosis. Via a standard posterolateral approach, the patient underwent a total hip arthroplasty, by a cemented 


\section{Orthopedics and Rheumatology Open Access Journal (OROAJ)}

femoral and acetabular component. In order to fill the bone loss, we used a cemented reconstruction with the addition of a Kerboull reinforcement cross. With use of bone graft from the femoral head (Figure 4). Cultures obtained at the time of surgery were negative. Pathological examination confirmed osteonecrosis. She received twenty days of thrombo-embolic prophylaxis with low molecular weight heparin with Post-operative rehabilitation and fully weight-bearing mobilization immediately after the operation. At 12 months follow-up she had no pain over the left hip and could walk without sticks, the Postel Merle d'Aubigné (PMA) score was 17.

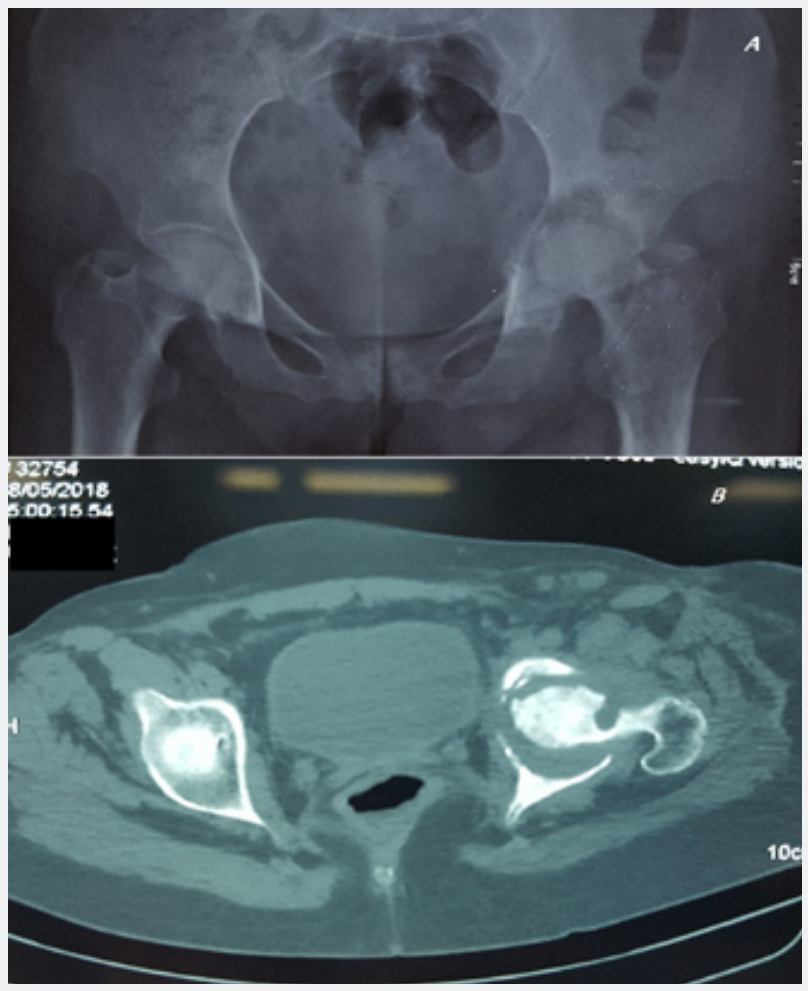

Figure 1:

A: radiograph showing an acetabular osteolysis, destruction of the left femoral head and pathological central dislocation of the hip. B: Computed Tomography revealing Osteolytic process of the acetabulum and femoral head destruction.
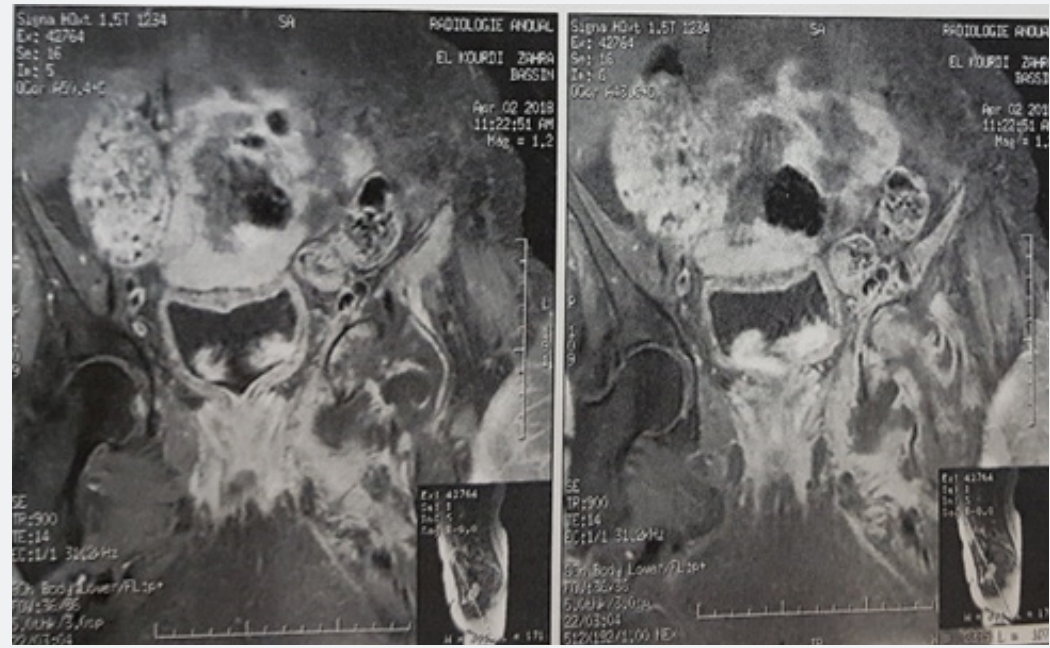

Figure 2: MRI of the pelvis showing osteonecrosis of the hip. 


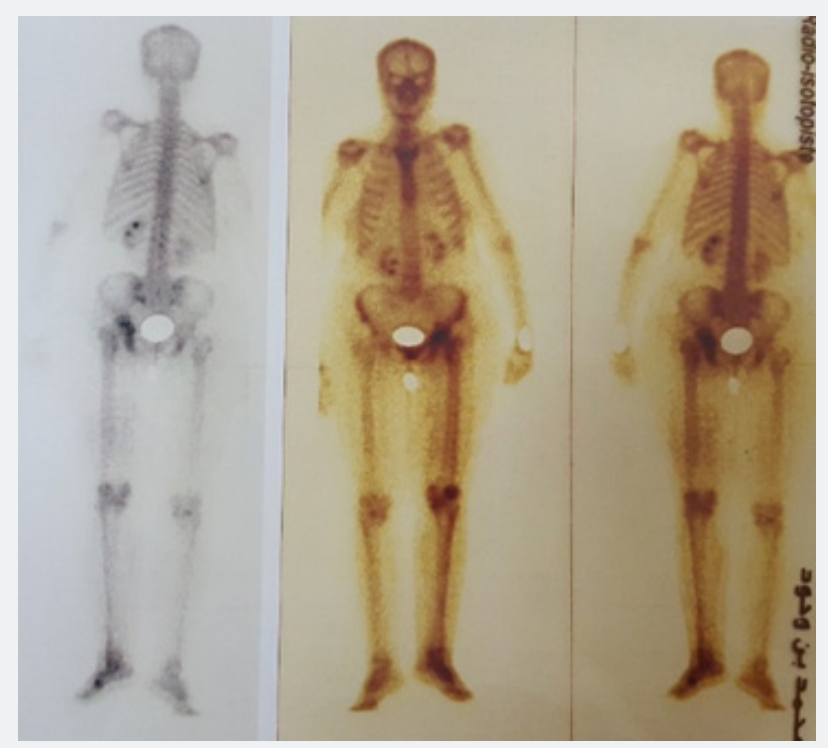

Figure 3: bone scan revealing increased uptake in the left acetabulum and femoral head.

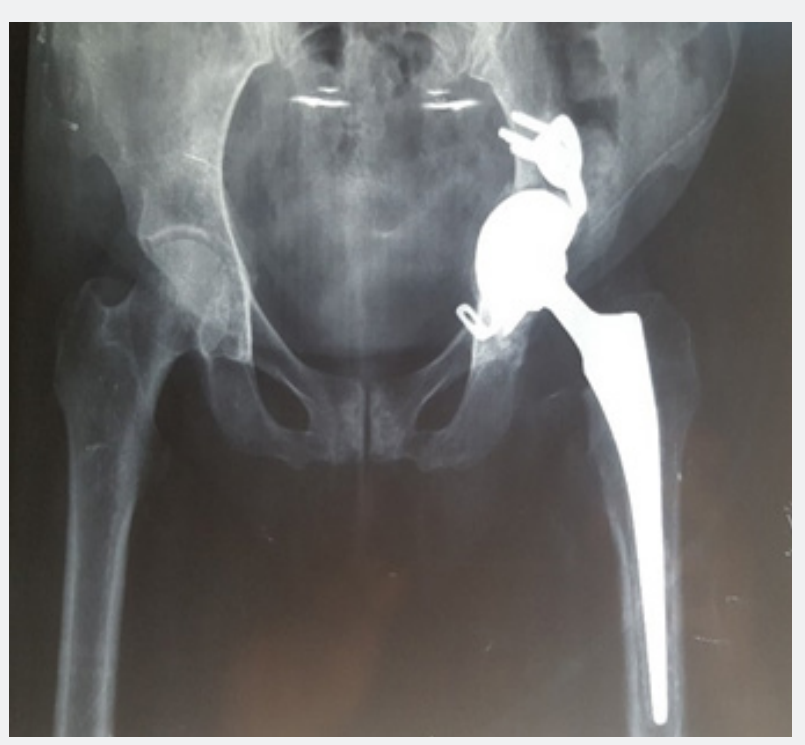

Figure 4: Postoperative control x-ray showing THA with a Kerboull reinforcement cross.

\section{Case 2}

A 50-year-old woman diagnosed with cervical cancer. She underwent radical hysterectomy; Adjuvant radiotherapy was given for the whole pelvis in April 2015. The exact dose distribution within the pelvis is not available. Two years after therapy, the patient presented to our department with bilateral hip pain which had been progressive and became worse recently. There was no history of fall, trauma or any significant injury prior to the onset of pain. Examination of the hips revealed painful movements, with restriction in the range of motion. The symptoms were more marked on the right side. She had no symptoms of infection and could walk less than one block. The rest of the general examination was normal.

Radiographs showed destruction of both femoral heads and osteolysis of the right acetabulum (Figure 5a). Computed tomography revealed no evidence of infection, bone metastasis, tumour recurrence, or radiation sarcoma (Figure 5b). The CT-guided biopsy revealed no evidence of malignancy in the right hip. The patient underwent a total hip arthroplasty by a cemented femoral and acetabular component. Pathological examination revealed no signs of malignancy. At eight months follow-up she had no pain over the right hip and could walk without sticks, the Postel Merle d'Aubigné (PMA) score was 15. 


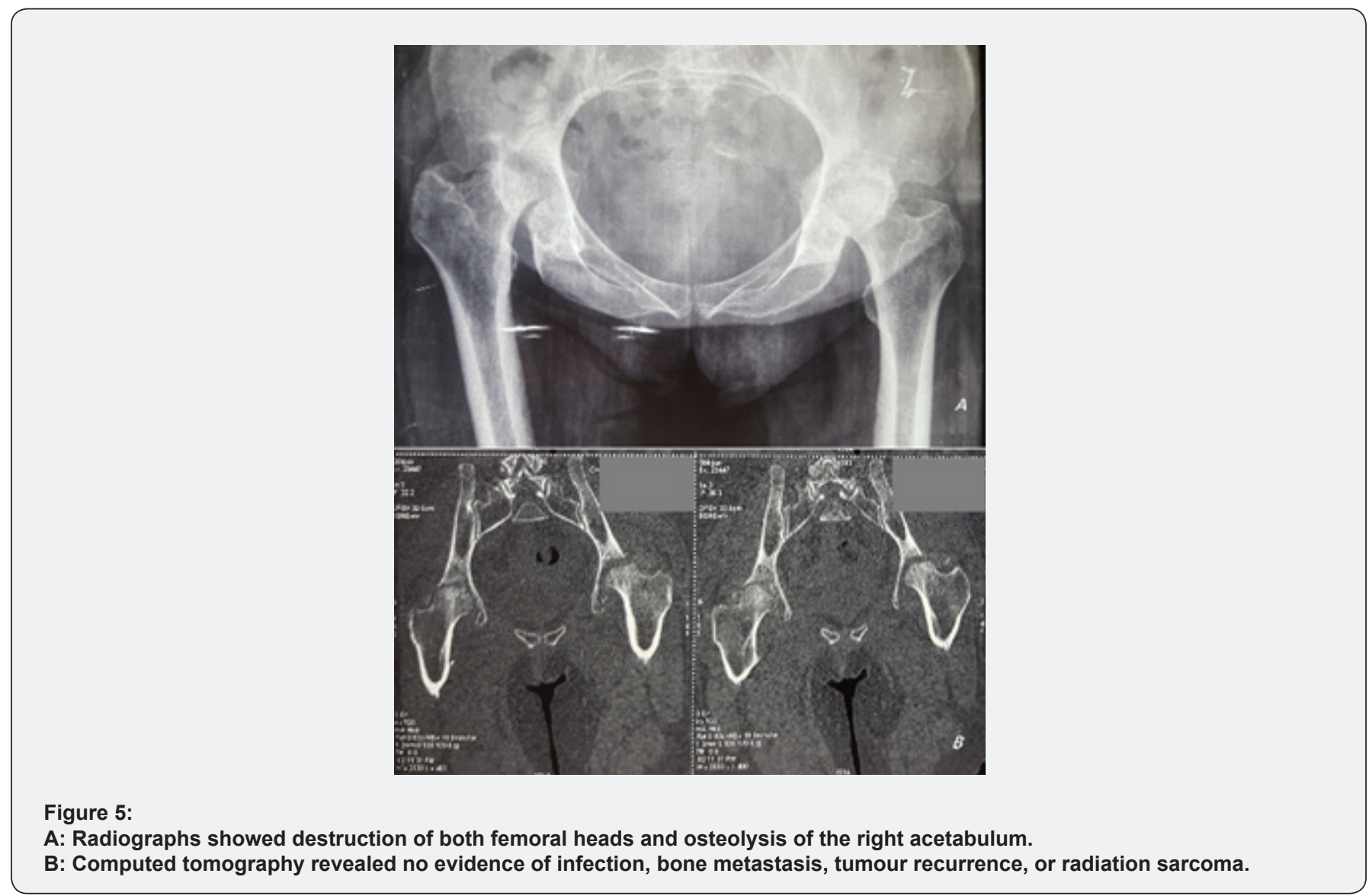

\section{Discussion}

Radiotherapy represents a highly effective and curative treatment modality for the treatment of pelvic malignancies and for metastatic bone disease [1]. Because of the anatomic vicinity of these cancers, the femoracetabular articulation can be exposed to a large dose of radiation that may induce changes in the skeletal system $[2,5,6]$. These injuries range in severity from radiation osteitis, through stress fractures and avascular osteonecrosis, to pathological fractures [1,3]. There are very few cases of radiation-induced hip necrosis described in the literature [4]. It is characterized by cellular death of bone components, due to the cellular depletion caused radiation and the local ischemia resulting from radiotherapy-induced microvascular damage $[2,4]$. These changes may be deleterious effects on osseointegration and provide a challenge for long-term implant stability in THA patients [7]. $0.44 \%$ of symptomatic osteoradionecrosis involve pelvic bones, and the median time of onset is 44 months [6]. Postirradiation lesions are often bilateral (21\%) [8]. In the first case, only the left hip was affected by osteonecrosis, perhaps resulting from a higher radiation dose on the left hip, or even inadequate shielding of the left hip during the radiotherapy.

Radiation-induced protrusion is very rare, the mechanism relates to the weight-bearing forces operatives in the hip, and multiple insufficiency type stress fractures superimposed upon the diminished structural strength occurring during bone revascularization and remodeling [9].

The probability of radiation induced changes in bone depends on many factors, such as dose per fraction, total dose, dose intensity and irradiated volume [2]. The reported incidence of pelvic Osteoradionecrosis varies widely, with a range of 2.1-34 \% depending on technique, criteria applied and many factors, such as dose per fraction, total dose, dose intensity and irradiated volume [2]. The extent on bone depends on patient-related factors such as age, sex, body weight, skeletal co-morbidities and co-medications, primarily corticosteroids [3]. Osteoradionecrosis constitutes a very difficult diagnostic problem, as the symptoms often appear many years after radiotherapy and patients do not associate them with past treatment $[2,8]$. It is important to differentiate osteoradionecrosis from local recurrence of the malignancy, bone metastasis, radiation induced sarcoma, and infection, especially in patients with a history of malignancy [6]. While computed tomography (CT) plays an important role in fracture detection, magnetic resonance imaging (MRI) is more sensitive to bone marrow abnormalities and to evaluate the viability of the femoral head [3-10]. Bone scan shows a typical symmetric uptake pattern in osteoradionecrosis, whereas metastasis appears asymmetrical $[6,11]$. 
THA in a patient with a post-irradiated pelvis remains a challenge [7]. Rates of early failure of traditional implants have been documented as high as $44 \%$ and $52 \%$ at 2-6 years, for both cemented and uncemented components due to dense sclerotic bone, and risk of infection du to damaged tissues that may provide a site for colonization following bacteremia [12]. In addition, host defense mechanisms may have been compromised by irradiationinduced damage and by the lymph stasis or lymphedema [5]. The failure of uncemented components is due to poorly elastic bone, reduced capacity of bone matrix to remodel over time and his decreased capability for osseous integration. The use of cemented cups with or without augmentation rings prevent early loss of fixation [12]. However, reported outcomes of THA have been disappointing [7]. The failure of cemented components is due to bad cement interdigitation and inability of bone to withstand the stress around the implant, microfractures are created inducing implant fails $[1,13]$.

The addition of a Kerboull reinforcement cross to the reconstruction reduce the risk of loosening of the acetabular component by giving elasticity to the implant, decreasing the stress applied to the peri-acetabular bone, and allows fixation over a large surface area [1-8]. The use of trabecular metal implants has shown better primary stability and bone ingrowth because of their high coefficient of friction and porosity $[1,14]$. Joglekar et al. [15] investigated outcomes of 22 hips under-going primary THA using tantalum acetabular cups in patients who had previously undergone pelvic radiotherapy. At 5-year follow-up, no patients presented signs of acetabular loosening [8].

\section{Conclusion}

The long-term side effects of radiotherapy depend on many factors, but the pathologic features are consistent. Although Osteoradionecrosis seems to be a very rare side-effect of radiotherapy, it may lead to severe functional impairment in patients who often have been cured of cancer. An early diagnosis and proper treatment may protect patients from long-term morbidities.

\section{References}

1. A Felden, G Vaz, S Kreps, P Anract, M Hamadouche, D J Biau (2015) A cemented acetabular component with a reinforcement cross provides excellent medium-term fixation in THA. Bone Joint J 97: 177-184.
2. Lukasz M, Dorota G, Roland k, Jerzy W, krystyna T (2011) Radiotherapy induced hip joint avascular necrosis: Two cases report. Rep Pract Oncol Radiother 16(5): 198-201.

3. Adrian J Meixel, Henrik Hauswald, Stefan Delorme, Bjorn Jobke (2018) From radiation osteitis to osteoradionecrosis: incidence and MR morphology of radiation-induced sacral pathologies following pelvic radiotherapy. Eur Radiol 28(8): 3550-3559.

4. Abdulkareem IH (2013) Radiation-induced femoral head necrosis. Niger J Clin Pract 16(1): 123-126.

5. Yang SH, Rong-Sen Yang, Chin-Lin Tsai (2001) Septic arthritis of the hip joint in cervical cancer patients after radiotherapy. Journal of Orthopaedic Surgery 9(2): 41-45.

6. Kwong-yin Chung, Kwok-hing Chiu, Kin-wing Cheung (2010) Osteoradionecrosis of the acetabulum in a total hip arthroplasty. J Orthop Surg 18(1): 110-112.

7. Mohit M Kukreja, Steven K Nishiyama, Parminder S Kang (2018) Management of radiation induced, bilateral Primary THA loosening with an aseptic Paprosky Type IIB acetabular defect and a contralateral septic Type IIIB acetabular defect: A case report and review of literature. Int J Surg Case Rep

42: 218-223.

8. Wodaje T, wretenberg P (2012) Long Term Effects of RadiationInduced Osteonecrosis of the Pelvis Caused Complete Failure of Total Hip Arthroplasty, Surgical Science 3(7): 373-375.

9. FM Hall, PM Mauch, MB Levene, MA Goldstein (1979) Prtrusio acetabulari following pelvic irradiation. AJR 132(2): 291-293.

10. Cabarrus MC, Ambekar A, Lu Y, Link TM (2008) MRI and CT of insufficiency fractures of the pelvis and the proximal femur. AJR Am J Roentgenol 191(4): 995-1001.

11. Peh WC, Khong PL, Yin Y, Ho WY, Evans NS, et al. (1996) Imaging of pelvic insufficiency fractures. Radiographics 16(2): 335-348.

12. P Massin, J Duparc (1995) Total hip replacement in irradiated hips: a retrospective study of 71 cases. J Bone Joint Surg Br 77(6): 847-852.

13. Kang-ll Kim, Gregg R Klein, loshua Sleeper, Adam P Dicker, Richard H Rothman, et al. (2007) Uncemented total hip arthroplasty in patients with a history of pelvic irradiaiion for prostate cancer. J Bone Joint Surg Am 89(4): 798-805.

14. Bobyn JD, Poggie RA, Krygier JJ, D G Lewallen, A D Hanssen, et al. (2004) Clinical validation of a structural porous tantalum biomaterial for adult reconstruction. J Bone Joint Surg 86: 123-129.

15. Siddharth B Joglekar, Peter S Rose, David G Lewallen, Franklin H Sim (2012) Tantalum acetabular cups provide secure fixation in THA after pelvic irradiation at minimum 5-yearfollowup. Clin Orthop Relat Res 470(11): 3041-3047. 
This work is licensed under Creative Commons Attribution 4.0 License

DOI: 10.19080/OROAJ.2021.17.555965
Your next submission with Juniper Publishers will reach you the below assets

- Quality Editorial service

- Swift Peer Review

- Reprints availability

- E-prints Service

- Manuscript Podcast for convenient understanding

- Global attainment for your research

- Manuscript accessibility in different formats ( Pdf, E-pub, Full Text, Audio)

- Unceasing customer service

Track the below URL for one-step submission https://juniperpublishers.com/online-submission.php 